\title{
Seeking predictors for paroxysmal atrial fibrillation in stroke with an online clinical database
}

\author{
Sefik Evren Erdener \\ Hacettepe University, Institute of Neurological Sciences and Psychiatry, Ankara, Turkey
}

\begin{abstract}
OBJECTIVE: A considerable fraction of ischemic stroke cases remain cryptogenic and there is increasing data suggesting the role of missed paroxysmal atrial fibrillations (PAF) in at least a number of these cases. Since electrophysiological identification of pAFs can be challenging, there has been an accumulation of proposed predictors and biomarkers for pAFs. The predictive values of these is varying and sometimes conflicting among studies. Therefore, we aimed to verify a fraction of previously reported parameters for pAF detection by investigating an independent clinical sample.

METHODS: Using a publicly available data downloaded from the MIMIC-3 intensive care unit database, we tested the predictive role of particular risk factors and biomarkers for pAF detection after ischemic stroke in 124 patients with ischemic stroke admitted within 24 hours of stroke onset.

RESULTS: Our evaluation revealed a strong association of older age in women, as well as admission National Institutes of Health Stroke Scale (NIHSS) and discharge modified Rankin Scores (mRS) in both sexes for pAFs, in patients that were in sinus rhythm on admission. We also detected a trend for lower gender-adjusted hemoglobin in patients with pAF, although the difference was insignificant. On the other hand, we did not find any significant association of pAF detection with some other previously reported biomarkers: serum magnesium level, leukocyte count, neutrophi/lymphocyte ratio or left atrial dilatation.

CONCLUSION: Even though our analysis did not reveal a strong and specific biomarker to predict pAFs after stroke, it identified key risk factors. It may be necessary to consider the possibility of pAFs and perform rigorous evaluation to prevent further events of embolic stroke in female patients older than 75 years, with more severe neurological deficits on admission, higher disability on discharge and also with relatively lower hemoglobin level. This first study from Turkey using clinical data from the MIMIC-3 database also demonstrates the value of publicized clinical data for confirmatory studies on various medical fields across the World.
\end{abstract}

Keywords: Clinical markers; database; embolism; paroxysmal atrial fibrillation; stroke.

Cite this article as: Erdener SE. Seeking predictors for paroxysmal atrial fibrillation in stroke with an online clinical database. North Clin Istanb 2020;7(4):378-385.

Tschemic stroke is a leading cause of death and disabil1 ity worldwide and is a heterogeneous phenomenon with a range of different etiological factors [1]. While in clinical practice, it can usually be identified as thrombotic or embolic nature, many cases remain cryptogenic with no obvious etiology to be determined [2,3]. Etiological identification is crucial for treatment decisions on sec- ondary prophylaxis to prevent recurrent stroke events. Atrial fibrillation (AF), either persistent or intermittent (i.e., paroxysmal) is one of the most common factors leading to cardioembolic stroke, especially in the elderly $[4,5]$. Many cryptogenic strokes are suspected to result from paroxysmal episodes of atrial fibrillation, and identification of AF can be critical as it shifts the stroke pro-

Received: March 26, 2019 Accepted: August 01, 2019 Online: December 05, 2019

Correspondence: Sefik Evren ERDENER, MD. Hacettepe Universitesi, Norolojik Bilimler ve Psikiyatri Enstitusu, Ankara, Turkey. 
phylaxis to an anticoagulation approach $[4,5]$. Confirming the presence of paroxysmal atrial fibrillation ( $\mathrm{pAF}$ ) can be challenging due to its temporary and sometimes short-lasting nature. Approximately half of patients with AF have no structural heart disease, and therefore echocardiography has limited value although left atrial dilatation has been associated with AF [6]. Most of the standard diagnostic procedures have low sensitivity for pAF detection [7]. Extended durations of ECG recording, sometimes up to several weeks, may be necessary for detection of some episodes $[8,9]$, but these resources are not widely available. Therefore, there are many attempts to identify novel and easy-to-test biomarkers for pAF [10]. These risk factors and biomarkers can at least identify a fraction of patients that need to be extensively evaluated for pAF episodes, allowing allocation of clinical resources more efficiently. A number of predictors, such as advanced age and female sex [11] or blood parameters like anemia [11, 12], iron deficiency [13], hyperuricemia [14-16], serum potassium and magnesium $[17,18]$, leukocyte count [19], neutrophil-to-lymphocyte ratio $[20,21]$ and vitamin-D level [22] have been associated with AF previously. Specifically for ischemic stroke patients, N-terminal Pro-Brain Natriuretic Peptide (BNP) levels have been reported to predict $\mathrm{pAF}$ episodes with high sensitivity and specificity [23]. However, there are also conflicting studies with some of these factors, like discrepant results on association of neutrophil/lymphocyte ratio and serum magnesium levels with $\operatorname{AF}[24,25]$. Thus, it can be useful to confirm the predictive value of previously proposed factors for pAF detection specifically for stroke patients acquired from an independent clinical population.

In this study, we aimed to test the predictive power of certain parameters for $\mathrm{pAF}$ detection in acute ischemic stroke patients utilizing an online clinical database. Medical Information Mart for Intensive Care-3 (MIMIC-3) is the updated version an intensive care unit research database freely open to the medical research community $[26,27]$. It was published in 2006 by the Computational Physiology Laboratory of the Massachusetts Institute of Technology (MIT), the Beth Israel Deaconess Medical Center (BIDMC) and the Philips Medical Center in the United States. A number of retrospective studies have been performed on this clinical dataset from several thousand intensive care unit (ICU) patients with multidisciplinary medical problems [28-34]. To our knowledge, our work is the first published study using data from stroke patients in MIMIC database. Besides being a confirmatory study for previously published predictors for AF, this work is an example for how these open-access clinical resources can be exploited for clinical research.

\section{MATERIALS AND METHODS}

The contents of the MIMIC database were downloaded from the Physionet website (http://physionet.org/physiobank/database/mimic $3 \mathrm{cdb}$ ) after receiving the necessary online trainings and signing the data use agreement. Ethical approval and consent to participate was not necessary and applicable for this study. The database contained clinical data, including medical history, physical examination, inpatient progress notes, diagnoses, laboratory and radiology results from 46520 ICU patients and 58976 admissions at the time it was accessed for this study. Diagnosis tables were searched to identify patients hospitalized for ischemic stroke, using keywords "stroke" "cerebrovascular accident" and "cva". Initial list returned 1021 admitted patients. After initial checks on clinical records, 169 cases were excluded since they had in fact other diagnoses such as subarachnoid hemorrhage, intraparenchymal hemorrhage or stroke mimics. The remaining 852 cases were randomized and 150 of them were selected for analysis. Patients younger than 50 years of age, admitted beyond the first 24 hours of stroke onset, with no evidence of major arterial occlusion or with arterial dissection were excluded ( 26 cases). Analyses proceeded for the remaining 124 patients.

The database provided details on stroke etiological evaluation, including but not limited to magnetic resonance imaging (with angiography), Doppler ultrasound, electrocardiography (ECG), continuous telemetric monitoring and echocardiography. Blood workup in all cases included complete blood count (CBC), renal function tests, lipid profiles and serum electrolytes. We included hemoglobin, hematocrit, erythrocyte and leukocyte counts, serum potassium, magnesium, calcium for predictive tests in our study since these measurements were available in all admitted cases. Other previously reported predictive parameters, like BNP, vitamin-D or uric acid were not tested or results were not reported in database, and therefore they could not be analyzed in this study.

\section{Statistical Analyses}

Analyses were performed using IBM SPSS Statistics 23 software. All data are reported as mean \pm standard de- 


\section{TABLE 1. Clinical characteristics of patients}

\begin{tabular}{|c|c|c|c|c|}
\hline & $\begin{array}{l}\text { No } A F \\
(N=51)\end{array}$ & $\begin{array}{c}A F \\
(n=73)\end{array}$ & $\mathrm{p}$ & OR \\
\hline Gender (Female) (\%) & 43.1 & 60.3 & 0.05 & 2.0 \\
\hline Age (male) & $70.6 \pm 7.8$ & $77.8 \pm 7.8$ & 0.002 & \\
\hline Age (female) & $772.4 \pm 11.2$ & $81 \pm 9.4$ & 0.02 & \\
\hline AF on admission (\%) & $\mathrm{N} / \mathrm{A}$ & 57.5 & $\mathrm{~N} / \mathrm{A}$ & $\mathrm{N} / \mathrm{A}$ \\
\hline Hypertension (\%) & 68.6 & 84.9 & 0.025 & 2.832 \\
\hline Diabetes (\%) & 39.2 & 24.6 & 0.114 & 0.516 \\
\hline Hyperlipidemia (\%) & 54.9 & 54.7 & 0.943 & 1.026 \\
\hline Coronary artery & & & & \\
\hline disease $(\%)$ & 35.3 & 39.7 & 0.535 & 1.265 \\
\hline Previous stroke (\%) & 13.7 & 17.8 & 0.620 & 1.410 \\
\hline LA dilatation (\%) & $\begin{array}{c}61.7 \\
(n=47)\end{array}$ & $\begin{array}{c}82.2 \\
(n=56)\end{array}$ & 0.026 & 2.857 \\
\hline $\begin{array}{l}\text { Intracranial } \\
\text { hemorrhage (\%) }\end{array}$ & 4 & 9.7 & 0.309 & 2.531 \\
\hline $\begin{array}{l}\text { Decompression } \\
\text { surgery }(\%)\end{array}$ & 4 & 1.4 & 0.567 & 0.338 \\
\hline Hospital infection (\%) & 15.5 & 30 & 0.128 & 2.197 \\
\hline $\begin{array}{l}\text { Hemoglobin } \\
\text { (male) }(\mathrm{g} / \mathrm{dL})\end{array}$ & $13.5 \pm 2.1$ & $12.9 \pm 2.2$ & 0.360 & \\
\hline $\begin{array}{l}\text { Hemoglobin } \\
\text { (female) }(\mathrm{g} / \mathrm{dL})\end{array}$ & $12.9 \pm 1.5$ & $12.3 \pm 1.7$ & 0.180 & \\
\hline Hematocrit (male) (\%) & $40.3 \pm 4.3$ & $38.6 \pm 5.7$ & 0.196 & \\
\hline Hematocrit (female) (\%) & $38.3 \pm 3.6$ & $37.5 \pm 4.0$ & 0.441 & \\
\hline $\mathrm{RBC}$ (male) $\times 10^{6} / \mathrm{mm}^{3}$ & $4.5 \pm 0.6$ & $4.2 \pm 0.7$ & 0.123 & \\
\hline RBC (female) $\times 10^{6} / \mathrm{mm}^{3}$ & $4.2 \pm 0.6$ & $4.2 \pm 0.5$ & 0.919 & \\
\hline WBC $\left(\times 10^{3} / \mathrm{mm}^{3}\right)$ & $10.2 \pm 4.1$ & $9.8 \pm 3.4$ & 0.626 & \\
\hline Neutrophil $\left(x 10^{3} / \mathrm{mm}^{3}\right)$ & $7.8 \pm 4.8$ & $7.8 \pm 3.4$ & 0.982 & \\
\hline Lymphocyte $\left(\times 10^{3} / \mathrm{mm}^{3}\right)$ & $1.7 \pm 0.8$ & $1.6 \pm 0.9$ & 0.538 & \\
\hline N/L Ratio & $5.9 \pm 5.1$ & $6.9 \pm 5.4$ & 0.464 & \\
\hline Potassium (mEq/L) & $4.1 \pm 0.5$ & $4.2 \pm 0.8$ & 0.712 & \\
\hline Magnesium (mg/dL) & $2.0 \pm 0.3$ & $1.9 \pm 0.3$ & 0.439 & \\
\hline $\begin{array}{l}\text { Calcium-(corrected) } \\
(\mathrm{mg} / \mathrm{dL})\end{array}$ & $9.2 \pm 0.6$ & $9.3 \pm 0.5$ & 0.817 & \\
\hline $\begin{array}{l}\text { SBP on admission } \\
(\mathrm{mmHg})\end{array}$ & $159 \pm 36$ & $156 \pm 26$ & 0.614 & \\
\hline DBP on admission & $79 \pm 18$ & $81+19$ & 0.534 & \\
\hline Admission NIHSS score & $11.6 \pm 5.6$ & $18.9 \pm 4.9$ & $<0.001$ & \\
\hline Discharge mRS & $3.9 \pm 1.4$ & $4.5 \pm 1.3$ & 0.013 & \\
\hline $\begin{array}{l}\text { Duration of } \\
\text { hospitalization (days) }\end{array}$ & $.9 \pm 11.1$ & $10.6 \pm 10.9$ & 0.442 & \\
\hline
\end{tabular}

NIHSS: National Institutes of Health Stroke Scale; mRS: Modified Rankin Score; SBP: Systolic blood pressure; DBP: Diastolic blood pressure; RBC: Red blood cell count; WBC: White blood cell count; N/L: Neutrophil/lymphocyte; LA: Left atrium; AF: Atrial fibrillation; OR: Odds ratio.
TABLE2. Classification of stroke etiology

\begin{tabular}{lcc} 
& No AF & AF \\
\hline Embolic & 16 & 72 \\
Atherosclerotic & 17 & 1 \\
Cryptogenic & 18 & None \\
Total & 51 & 73 \\
\hline
\end{tabular}

AF: Atrial fibrillation.

viation. Categorical variables were compared with ChiSquare tests and nominal variables were compared with $t$-tests across independent groups. The variables that passed the initial tests to show significant difference between $\mathrm{AF}$ and no-AF groups were further tested for their predictive potential on pAFs using binary logistic regression analyses with appropriate adjustments. $\mathrm{P} \leq 0.05$ was accepted as the cut-off for statistical significance.

\section{RESULTS}

Clinical characteristics of patients are presented in Table 1. Middle cerebral artery (MCA) was occluded in 103 cases $(83.0 \%)$, internal carotid artery (ICA) in 14 $(11.3 \%)$ while the remaining 7 patients had occlusions in anterior cerebral, posterior cerebral or basilar arteries. Mean hospitalization duration was $9.7+10.7$ days. All patients were admitted within 24 hours of stroke onset. 42 patients $(33.9 \%)$ had documented AF on admission. These patients also had AF rhythm in their subsequent ECGs indicating that they had persistent AF. On the other hand, telemetry monitoring revealed paroxysmal $\mathrm{AF}$ (pAF) in $31(25.0 \%)$ patients who initially had sinus rhythm in their ECGs. Hence, in total, 73 cases (58.9\%) had either persistent or paroxysmal AF documented during their hospital stay.

Etiological causes of stroke in these patients are shown in Table 2. Only one patient in AF group had a clearly atherosclerotic cause of ischemic stroke while others were classified as cardioembolic. In the group without AF (persistent or paroxysmal), 16 patients had other sources of cardioembolism while 17 patients had atherosclerotic stoke. Eighteen patients had no identifiable etiology and were classified as cryptogenic. It should be noted that if there was no telemetry monitoring, 31 patients with $\mathrm{pAF}$ would also have remained 
TABLE 3. Univariate logistic regression analysis in patients with no AF on admission

\begin{tabular}{lcccccc} 
& No pAF $(\mathrm{N}=51)$ & pAF $(\mathrm{n}=31)$ & $\mathrm{P}$ & OR & $95 \%$ CI & ROC curve- AUC \\
\hline Gender (female) $(\%)$ & 43.1 & 64.5 & 0.044 & 2.397 & $0.954-6.020$ & \\
Age (male) & $70.6 \pm 11.5$ & $77.6 \pm 9.3$ & 0.070 & 1.068 & $0.995-1.148$ & 0.686 \\
Age (female) & $72.4 \pm 11.2$ & $79.5 \pm 9.6$ & 0.035 & 1.068 & $1.002-1.140$ & 0.699 \\
LA dilatation (\%) & $29(61.7)(\mathrm{n}=47)$ & $17(65.4)(\mathrm{n}=26)$ & 0.805 & 1.172 & $0.431-3.184$ & \\
Hypertension (\%) & 68.6 & 83.9 & 0.131 & 2.375 & $0.771-7.299$ & \\
Hemoglobin (male) (g/dL) & $13.5 \pm 2.1$ & $13 \pm 2.5$ & 0.571 & 0.895 & $0.653-1.225$ & 0.535 \\
Hemoglobin (female) (g/dL) & $12.9 \pm 1.5$ & $12.4 \pm 1.5$ & 0.304 & 0.798 & $0.521-1.222$ & 0.657 \\
Admission NIHSS score & $11.7 \pm 5.6$ & $17 \pm 4.9$ & 0.01 & 1.219 & $1.029-1.443$ & 0.767 \\
Discharge mRS & $3.86 \pm 1.4$ & $4.6 \pm 0.9$ & 0.02 & 1.567 & $1.054-2.331$ & 0.616 \\
\hline
\end{tabular}

NIHSS: National Institutes of Health Stroke Scale; mRS: Modified Rankin Score; pAF: Paroxysmal atrial fibrillation; OR: Odds ratio; CI: Confidence interval; ROC: Receiver operating characteristic; AUC: Area under the curve.

cryptogenic. In patients with AF (both persistent and paroxysmal), we detected a significantly higher fraction of female sex, hypertension, left atrial dilatation, as well as older age, admission National Institutes of Health Stroke Scale (NIHSS) score and modified Rankin Score (mRS) (Table 1). There were no significant differences in leukocyte counts, lymphocyte/neutrophil ratios, serum potassium or magnesium levels between $\mathrm{AF}$ and no-AF groups, unlike a number of previous studies $[17,18,21,35]$. Meanwhile, we detected a relatively lower hemoglobin levels after adjusting for sex in line with previous work [11] although the difference in our analysis was not significant.

Next, focusing on the parameters showing difference between AF and non-AF groups, we tested the predictive potential of those parameters for subsequent $\mathrm{pAFs}$ in patients with sinus rhythm on admission $(n=82)$. These results are shown in Table 3. Univariate logistic regression revealed a significant association of $\mathrm{pAF}$ detection with patient age in females $(\mathrm{OR}=1.068$, $\mathrm{p}=0.035)$ and a near-significant association in males $(\mathrm{OR}=1.068, \mathrm{p}=0.07)$. Receiver operating characteristic (ROC) analysis showed a cut-off of 75 for females for $80 \%$ Sensitivity and $73 \%$ Specificity (Area under the curve $(A U C)=0.699)$ (Fig. 1A, B). pAFs were more than twice as common in female patients (OR 2.562, $\mathrm{p}=0.044)$. The effect of gender on $\mathrm{pAF}$ detection was dependent on age, however (Fig. 1C). Despite the lack of significance between ages of men and women (Fig. 1C), age-adjusted sex effect on pAF detection was not significant in logistic regression analy- sis either, $(p=0.126)$, supporting the contributing role of age on sex-related differences. After adjustments for age and sex, patients with pAF tended to have a significantly higher NIHSS score on admission $(\mathrm{OR}=1.222$, $\mathrm{p}=0.025 ; \mathrm{AUC}=0.767)$ and nearly-significantly higher $\mathrm{mRS}(\mathrm{OR}=1.464, \mathrm{p}=0.080)$ on discharge although age was also found as a contributing factor for higher $\mathrm{mRS}$ (Pearson coefficient: 0.314, $\mathrm{p}=0.004$ ). ROC curve analysis for NIHSS scores showed a $70 \%$ sensitivity and $61 \%$ specificity for a cut-off level of 13 (AUC=0.767) (Fig. 1D).On the other hand, correlation analysis was significant between age and admission NIHSS (Pearson coefficient: $0.216, p=0.251$ ), signifying that, unlike the disability score of $\mathrm{mRS}$, the symptomatic score of NIHSS was independent from age and sex.

Sex-adjusted hemoglobin levels again had a tendency to be lower in pAF groups, but the association did not reach statistical significance. At least in females, a contributing effect of hemoglobin on pAF cannot be excluded, and this is independent from age as hemoglobin level did not correlate with age in females (Pearson coefficient: $0.064, p=0.689)$. In males, hemoglobin level was significantly correlated with older age (Pearson coefficient: $-0.324, p=0.042)$. Left atrial dilatation, in contrast, was not significantly different between $\mathrm{pAF}$ and non-pAF groups, again conflicting with previous reports [36]. This finding supports the view that LA diameter primarily serves as a marker of persistent AF [6]. Finally, past medical history of hypertension was also not significantly associated with pAF detection $(\mathrm{p}=0.157)$, another factor differing from persistent $\mathrm{AF}$. 

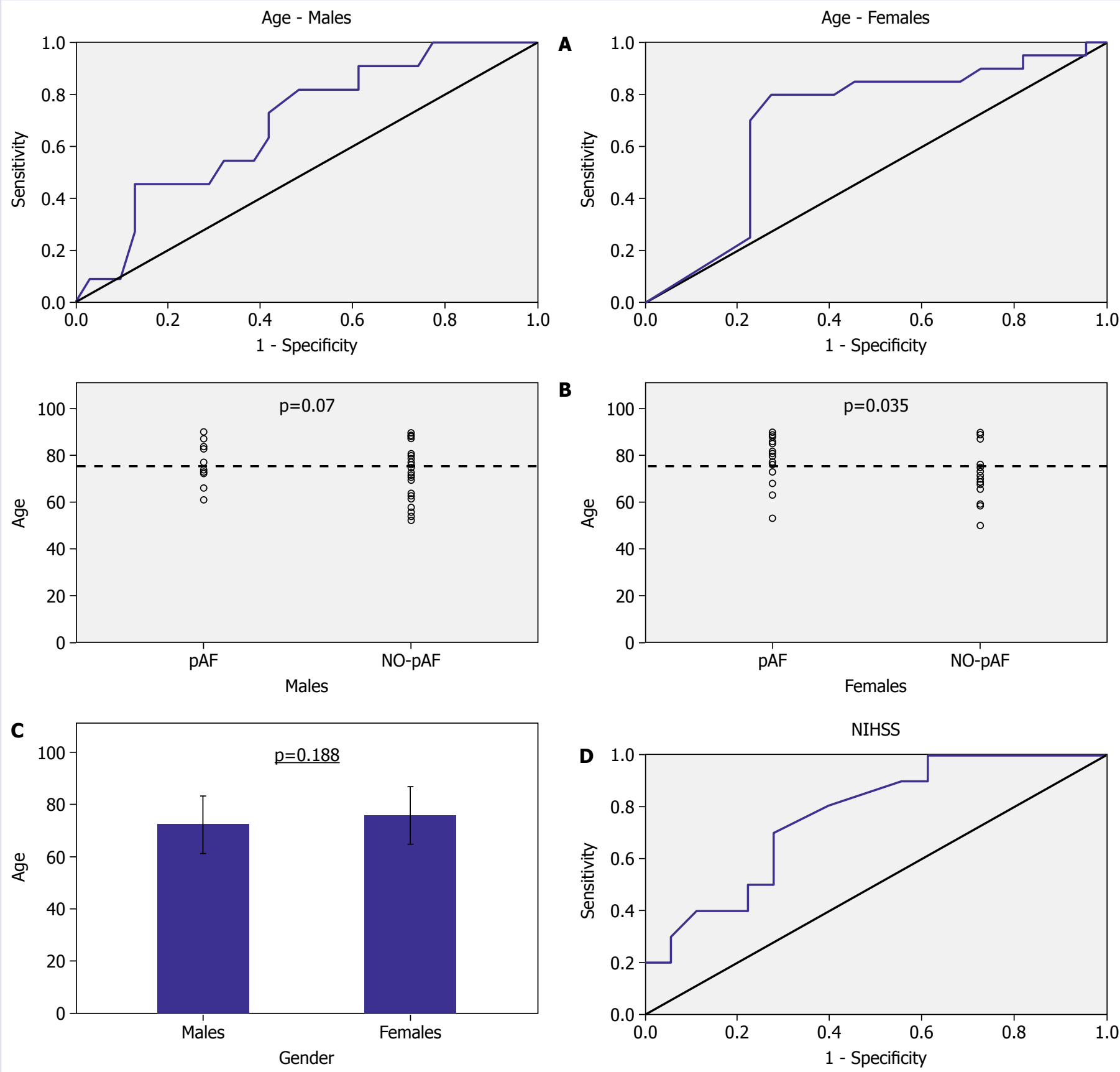

FIGURE 1. (A) Receiver operating characteristic (ROC) curve of age in males and females for pAF detection in ischemic stroke patients. Area under the curve (AUC) was 0.686 and 0.699 for males and females, respectively. For female patients, the cut-off value of 75 had $80 \%$ sensitivity and $73 \%$ specificity for pAF detection. (B) In the scatter plots, the discrimination between pAF and no-pAF groups with a cut-off age of 75 (dashed line) is evident in female patients. (C) Patient age was not significantly different across both genders, being $72.5 \pm 11.3$ and $75.8 \pm 11.0$ years in males and females, respectively $(p=0.188)$. (D) ROC curve of admission NIHSS score for pAF detection. AUC was 0.767 and a cut-off level of 13 showed a $70 \%$ sensitivity and $61 \%$ specificity.

\section{DISCUSSION}

This study evaluated the predictive potential of a number of previously introduced serum biomarkers and risk factors for $\mathrm{AF}$ and $\mathrm{pAF}$ utilizing an online database from an independent clinical population. Specifically, we tested the readily available parameters in stroke patients hospitalized within 24 hours of onset to predict subse- 
quent paroxysmal atrial fibrillations. This is a significant clinical problem because a prominent number of stroke cases remain cryptogenic for etiology with no apparent causative factors despite rigorous evaluation. Detection of a single pAF episode in that setting can influence the lifelong treatment decisions as anticoagulation would be mandatory to prevent recurrent strokes. It may be necessary to acquire long-term continuous ECG monitoring to catch electrophysiologic evidences of pAFs, which is not always possible in routine clinical practice. Therefore, identification of high-risk patients based on clinical factors or biomarkers would be extremely helpful to focus advanced investigations on these cases rather than all cryptogenic strokes.

Our work showed the predictive potential of age, admission NIHSS and discharge MRS to predict pAFs (as detected by telemetric monitoring) in patients with ischemic stroke hospitalized within 24 hours of onset and with no AF on admission. The effect of age on $\mathrm{pAF}$ detection is augmented in females, possibly due to agedependent sex-specific biological effects, like estrogen levels $[37,38]$. Serum biochemical or hematological parameters, like magnesium, potassium and leukocyte counts or neutrophil/lymphocyte ratios had no significant association with pAF. Also, although the difference was not significant in our analysis, it would be helpful to consider hemoglobin level during clinical evaluation as relatively lower hemoglobin level is usually found in pAF groups being neither confounded by age or gender. This association can be better evaluated in larger samples with higher statistical power. Overall, our results suggest that especially in female patients over 75 years of age with an admission NIHSS over 13, a pAF episode within approximately 10 days after stroke onset was very likely, which could be missed if no ECG monitoring had been performed. $83 \%$ of such cases had $\mathrm{pAF}$ in our patient population.

Atrial fibrillations are definitive causative factors for ischemic stroke [4], especially if detected before stroke [39], and it is highly recommended to include appropriate cardiac evaluation in the clinical work-up of stroke patients. However, one has to be aware of a possible overestimation of their role in recurrent strokes since a number of recent studies suggest that atrial fibrillation detected in the immediate post-stroke setting (either persistent or paroxysmal) could rather arise from a neurogenic autonomic dysregulation, questioning the cause-effect relationship of atrial fibrillations and stroke in such cases $[39,40]$. Future studies need to address the problem of neurogenic versus cardiogenic AFs to validate their association with recurrent stroke events when not anticoagulated, and to identify biochemical or structural cardiac markers to distinguish those two groups clinically. In addition to a neurogenic autonomic dysregulation, electrolyte imbalances, especially abnormalities in potassium and magnesium [17, $18,41]$, triggered after the stroke onset may also predispose the patients to arrhythmic events, including atrial fibrillations. As noted above, we did not find an association between such electrolyte abnormalities and the presence of AF in our sample.

Lack of association for potassium and magnesium levels or leukocyte parameters can arise for a number of reasons. First of all, this clinical database was prepared from the records of multiple institutions in the USA, and laboratory procedural standards might differ among hospitals, affecting the absolute values reported. Racial information was not systematically provided in those databases, but it can be a prominent confounder for blood measurements, and it is likely that data from different racial populations were pooled in this patient sample considering the residence of multiple ethnic groups in North America. Although the patients were monitored during their hospitalization for pAFs, it is possible that some very brief episodes were missed. Moreover, none of the patients were reported to have a Holter monitoring during inpatient phase or after discharge. Therefore, it is likely that a number of pAF cases were missed and still in the cryptogenic stroke group, which can result in this such discrepancies.

Despite the lack of an identified specific biomarker according to our study to predict pAF episodes, it has been helpful to confirm certain risk factors like sex, age, admission NIHSS and other contributing factors like hemoglobin level in an independent clinical sample. Ambitious long-term ECG monitoring in cryptogenic stroke cases can be beneficial especially for such high-risk groups. This work serves as the first study on stroke patients of MIMIC database, at least to our knowledge, and also underlines the value of online clinical databases for evaluation of predictive role of certain factors for specific patient populations. There has been a progressive accumulation of suggested biomarkers for $\mathrm{pAF}$ and other stroke etiologies over the last several years and it would be helpful to test novel markers in such independent clinical databases to confirm their predictive potential to build a collective medical research environment. 


\section{Conclusion}

There is a higher probability of pAFs after stroke, which may be the etiological cause, especially in older females admitted with higher NIHSS scores and discharged with higher disability. Additionally, low hemoglobin level can potentially be helpful for consideration of patients selected for advanced electrophysiologic evaluation. This study is a prime example of the utilization of public clinical databases for independent testing of various biomarkers identified in other clinical populations.

Acknowledgements: The author would like to thank Ellen Witkowski, Ph.D. (Boston University, USA) for English language editing of the manuscript.

Conflict of Interest: No conflict of interest was declared by the author.

Financial Disclosure: The author declared that this study has received no financial support.

\section{REFERENCES}

1. Hankey GJ, Warlow CP. Treatment and secondary prevention of stroke: evidence, costs, and effects on individuals and populations. Lancet 1999;354:1457-63. [CrossRef]

2. Kolominsky-Rabas PL, Weber M, Gefeller O, Neundoerfer B, Heuschmann PU. Epidemiology of ischemic stroke subtypes according to TOAST criteria: incidence, recurrence, and long-term survival in ischemic stroke subtypes: a population-based study. Stroke 2001;32:273540. [CrossRef]

3. Adams HP Jr, Bendixen BH, Kappelle LJ, Biller J, Love BB, Gordon DL, et al. Classification of subtype of acute ischemic stroke. Definitions for use in a multicenter clinical trial. TOAST. Trial of Org 10172 in Acute Stroke Treatment. Stroke 1993;24:35-41. [CrossRef]

4. Lin HJ, Wolf PA, Kelly-Hayes M, Beiser AS, Kase CS, Benjamin EJ, et al. Stroke severity in atrial fibrillation. The Framingham Study. Stroke 1996;27:1760-4. [CrossRef]

5. Saxonhouse SJ, Curtis AB. Risks and benefits of rate control versus maintenance of sinus rhythm. Am J Cardiol 2003;91:27D-32D. [CrossRef]

6. Suarez GS, Lampert S, Ravid S, Lown B. Changes in left atrial size in patients with lone atrial fibrillation. Clin Cardiol 1991;14:652-6.

7. Thomas G, Lerman BB. Prediction of stroke risk in atrial fibrillation, prevention of stroke in atrial fibrillation, and the impact of long-term monitoring for detecting atrial fibrillation. Curr Atheroscler Rep 2011;13:290-7. [CrossRef]

8. Alves M, Narciso MR, Cruz J, Rocha M, Fonseca T. Paroxysmal atrial fibrillation detection in patients with acute ischemic stroke through prolonged Holter: prospective study. Aging Clin Exp Res 2019;31:469-74.

9. Philippsen TJ, Christensen LS, Hansen MG, Dahl JS, Brandes A. Detection of Subclinical Atrial Fibrillation in High-Risk Patients Using an Insertable Cardiac Monitor.JACC Clin Electrophysiol 2017;3:1557-64.

10. Lind L, Sundström J, Stenemo M, Hagström E, Ärnlöv J. Discovery of new biomarkers for atrial fibrillation using a custom-made proteomics chip. Heart 2017;103:377-82. [CrossRef]

11. Distelmaier K, Maurer G, Goliasch G. Blood count in new onset atrial fibrillation after acute myocardial infarction - a hypothesis generating study. Indian J Med Res 2014;139:579-84.

12. Lee WH, Hsu PC, Chu CY, Lee HH, Lee MK, Lee CS, et al. Anemia as an Independent Predictor of Adverse Cardiac Outcomes in Patients with Atrial Fibrillation. Int J Med Sci 2015;12:618-24. [CrossRef]

13. Keskin M, Ural D, Altay S, Argan O, Börklü EB, Kozan Ö. Iron deficiency and hematinic deficiencies in atrial fibrillation: A new insight into comorbidities. Turk Kardiyol Dern Ars 2018;46:103-10. [CrossRef]

14. Huang G, Xu RH, Xu JB, Liu Y, Liu ZH, Xie X, et al. Hyperuricemia is associated with atrial fibrillation prevalence in very elderly - a community based study in Chengdu, China. Sci Rep 2018;8:12403. [CrossRef]

15. Memetoglu ME, Kehlibar T, Yilmaz M, Günay R, Arslan Y, Tuygun A, et al. Serum uric acid level predicts new-onset atrial fibrillation after coronary artery bypass graft operation. Eur Rev Med Pharmacol Sci 2015;19:784-9.

16. Zhang CH, Huang DS, Shen D, Zhang LW, Ma YJ, et al. Association Between Serum Uric Acid Levels and Atrial Fibrillation Risk. Cell Physiol Biochem 2016;38:1589-95. [CrossRef]

17. Khan AM, Lubitz SA, Sullivan LM, Sun JX, Levy D, Vasan RS, et al. Low serum magnesium and the development of atrial fibrillation in the community: the Framingham Heart Study. Circulation 2013;127:338. [CrossRef]

18. Krijthe BP, Heeringa J, Kors JA, Hofman A, Franco OH, Witteman JC, et al. Serum potassium levels and the risk of atrial fibrillation: the Rotterdam Study. Int J Cardiol 2013;168:5411-5. [CrossRef]

19. Weymann A, Ali-Hasan-Al-Saegh S, Sabashnikov A, Popov AF, Mirhosseini SJ, Liu T, et al; Surgery And Cardiology-Group ImcscGroup IMOC. Prediction of New-Onset and Recurrent Atrial Fibrillation by Complete Blood Count Tests: A Comprehensive Systematic Review with Meta-Analysis. Med Sci Monit Basic Res 2017;23:179222. [CrossRef]

20. Afari ME, Bhat T. Neutrophil to lymphocyte ratio (NLR) and cardiovascular diseases: an update. Expert Rev Cardiovasc Ther 2016;14:5737. [CrossRef]

21. Walsh KJ, Tan KS, Zhang H, Amar D. Neutrophil-lymphocyte ratio and risk of atrial fibrillation after thoracic surgery. Interact Cardiovasc Thorac Surg 2017;24:555-9. [CrossRef]

22. Belen E, Aykan AC, Kalaycioglu E, Sungur MA, Sungur A, Cetin M. Low-Level Vitamin D Is Associated with Atrial Fibrillation in Patients with Chronic Heart Failure. Adv Clin Exp Med 2016;25:51-7. [CrossRef]

23. Fonseca AC, Brito D, Pinho e Melo T, Geraldes R, Canhão P, Caplan LR, et al. $\mathrm{N}$-terminal pro-brain natriuretic peptide shows diagnostic accuracy for detecting atrial fibrillation in cryptogenic stroke patients. Int J Stroke 2014;9:419-25. [CrossRef]

24. Cook RC, Yamashita MH, Kearns M, Ramanathan K, Gin K, Humphries KH. Prophylactic magnesium does not prevent atrial fibrillation after cardiac surgery: a meta-analysis. Ann Thorac Surg 2013;95:53341. [CrossRef]

25. Celik AI, Kanadasi M, Demir M, Deniz A, Akilli RE, Deveci OS, et al. Predictors of the paroxysmal atrial fibrillation recurrence following cryoballoon-based pulmonary vein isolation: Assessment of left atrial volume, left atrial volume index, galectin-3 level and neutrophil-to-lymphocyte ratio. Indian Pacing Electrophysiol J 2019;19:9-14. [CrossRef]

26. Goldberger AL, Amaral LA, Glass L, Hausdorff JM, Ivanov PC, Mark RG, et al. PhysioBank, PhysioToolkit, and PhysioNet: components of a new research resource for complex physiologic signals. Circulation 2000;101:E215-20. [CrossRef]

27. Johnson AE, Pollard TJ, Shen L, Lehman LW, Feng M, Ghassemi M, et al. MIMIC-III, a freely accessible critical care database. Sci Data 2016;3:160035. [CrossRef] 
28. Ghassemi M, Marshall J, Singh N, Stone DJ, Celi LA. Leveraging a critical care database: selective serotonin reuptake inhibitor use prior to ICU admission is associated with increased hospital mortality. Chest 2014;145:745-52. [CrossRef]

29. Liang Y, Chen Z, Ward R, Elgendi M. Hypertension Assessment via ECG and PPG Signals: An Evaluation Using MIMIC Database. Diag. nostics (Basel) 2018;8:65. [CrossRef]

30. Zhao Y, Zhou H, Tan W, Song Y, Qiu Z, Li S, et al. Prolonged dexmedetomidine infusion in critically ill adult patients: a retrospective analysis of a large clinical database Multiparameter Intelligent Monitoring in Intensive Care III. Ann Transl Med 2018;6:304. [CrossRef]

31. Han YQ, Zhang L, Yan L, Li P, Ouyang PH, Lippi G, et al. Red blood cell distribution width predicts long-term outcomes in sepsis patients admitted to the intensive care unit. Clin Chim Acta 2018;487:112-6.

32. Vincent JL, Nielsen ND, Shapiro NI, Gerbasi ME, Grossman A, Doroff $\mathrm{R}$, et al. Mean arterial pressure and mortality in patients with distributive shock: a retrospective analysis of the MIMIC-III database. Ann Intensive Care 2018;8:107. [CrossRef]

33. Liu Q, Yang J, Zhang J, Zhao F, Feng X, Wang X, et al. Description of Clinical Characteristics of VAP Patients in MIMIC Database. Front Pharmacol 2019;10:62. [CrossRef]

34. Li K, Feng C, Jia L, Chen L, Pan F, Li T. Discussion of the implementation of MIMIC database in emergency medical study. [Article in Chinese]. Zhonghua Wei Zhong Bing Ji Jiu Yi Xue 2018;30:494-6.
35. Cosansu K, Vatan MB, Gunduz H, Akdemir R. Use of neutrophil-lymphocyte ratio for risk stratification and relationship with time in therapeutic range in patients with nonvalvular atrial fibrillation: A pilot study. Clin Cardiol 2018;41:339-42. [CrossRef]

36. Baturova MA, Sheldon SH, Carlson J, Brady PA, Lin G, Rabinstein AA, et al. Electrocardiographic and Echocardiographic predictors of paroxysmal atrial fibrillation detected after ischemic stroke. BMC Cardiovasc Disord 2016;16:209. [CrossRef]

37. Wong JA, Rexrode KM, Sandhu RK, Moorthy MV, Conen D, Albert CM. Menopausal age, postmenopausal hormone therapy and incident atrial fibrillation. Heart 2017;103:1954-61. [CrossRef]

38. Odening KE, Deiß S, Dilling-Boer D, Didenko M, Eriksson U, Nedios $\mathrm{S}$, et al6. Mechanisms of sex differences in atrial fibrillation: role of hormones and differences in electrophysiology, structure, function, and remodelling. Europace 2019;21:366-76. [CrossRef]

39. Sposato LA, Cerasuolo JO, Cipriano LE, Fang J, Fridman S, Paquet $\mathrm{M}$, et al; PARADISE Study Group. Atrial fibrillation detected after stroke is related to a low risk of ischemic stroke recurrence. Neurology 2018;90:e924-31. [CrossRef]

40. Sposato LA, Riccio PM, Hachinski V. Poststroke atrial fibrillation: cause or consequence? Critical review of current views. Neurology 2014;82:1180-6. [CrossRef]

41. Hayıroğlu Mİ, Keskin M, Kozan Ö. Electrolyte Imbalances as a predisposing factor for arrhythmias. Turk Kardiyol Dern Ars 2017;45:772. 